\title{
Análisis de las causas, características y consecuencias de la reexposición al fármaco o compuesto responsable de un episodio de hepatotoxicidad
}

\author{
A. Fernández-Castañer, M. García-Cortés, M. I. Lucena¹, Y. Borraz¹, G. Peláez², J. Costa ${ }^{3}$, S. Anzola ${ }^{3}$, \\ J. Salmerón ${ }^{4}$, S. Ávila ${ }^{5}$, J. A. Durán ${ }^{6}$, A. Malcón de Dios ${ }^{6}$, M. Romero-Gómez ${ }^{7}$, A. Madrazo , \\ T. Muñoz-Yagüe ${ }^{8}$ y R. J. Andrade
}

Servicios de Aparato Digestivo y ${ }^{I}$ Farmacología Clínica. Hospital Universitario Virgen de La Victoria. Facultad de Medicina. Málaga. ${ }^{2}$ Servicio de Aparato Digestivo. Hospital Torrecárdenas. Almería. ${ }^{3}$ Unidad de Farmacología y Servicio de Aparato Digestivo. Hospital Germans Trias i Pujol. Barcelona. ${ }^{4}$ Servicio de Aparato Digestivo. Hospital Clínico San Cecilio. Granada. ${ }^{5}$ Servicio de Aparato Digestivo. Hospital Xeral-Calde. Lugo ${ }^{6}$ Servicio de Farmacología Clínica. Hospital Virgen de la Macarena. Sevilla. ${ }^{7}$ Unidad de Hepatología. Hospital Universitario Valme. Sevilla. ${ }^{8}$ Servicio de Aparato Digestivo. Hospital 12 de Octubre. Madrid

\section{RESUMEN}

Introducción: la reexposición al agente causal constituye un incidente potencialmente grave en hepatotoxicidad.

Objetivos: evaluar las características y la evolución de los casos con reexposición positiva.

Material y métodos: estudio retrospectivo de una serie de casos con evidencia de reexposición positiva incluidos en el Registro Español de Hepatopatías Asociadas a Medicamentos, analizando su relación con variables demográficas y clínicas, causalidad, evolución y consecuencias.

Resultados: de un total de 520 casos, 31 (6\%) cumplían los criterios de reexposición. La evolución fatal, la necesidad de hospitalización y el tiempo medio de recuperación fueron mayores en la lesión tóxica de tipo hepatocelular. El grupo farmacológico identificado con mayor frecuencia fue el de los antibióticos. En la mayoría de los casos la reexposición con el compuesto responsable fue inadvertida (73\%) debido a: la ausencia de diagnóstico del caso índice, la carencia de información al paciente o a su médico y también (12\%) por el desarrollo de una reacción cruzada entre fármacos estructuralmente similares.

Conclusiones: la reexposición accidental a un mismo fármaco o a otro estructuralmente relacionado tras un primer episodio de hepatotoxicidad no es infrecuente y sus consecuencias pueden ser graves, especialmente en el tipo de lesión hepatocelular. Una minuciosa historia clínica y la sospecha diagnóstica reflejada en el informe del primer episodio podrían disminuir la incidencia de este evento iatrogénico.

Palabras clave: Reexposición. Hepatotoxicidad. Enfermedad hepática inducida por fármacos. Efectos adversos hepáticos.

\begin{abstract}
Introduction: reexposure to a causal agent represents a potentially serious event in hepatotoxicity.

Objectives: to assess the characteristics and outcome of cases with positive reexposure.

Material and methods: a retrospective study of cases with evidence of positive reexposure included in Registro Español de Hepatopatías Asociadas a Medicamentos, and an analysis of their relation to demographic and clinical variables, causality, course, and consequences.

Results: of a total of 520 cases $31(6 \%)$ met reexposure criteria. Fatal outcomes, needs for admission, and mean recovery time were all higher for hepatocellular-type toxic injury. The most commonly identified drug class was antibiotics. On most occasions $(73 \%)$ reexposure to the causal compound escaped notice because of: absence of index case diagnosis, lack of information to patients and their physicians, and (12\%) development of cross reactions between structurally similar drugs.

Conclusions: accidental reexposure to a drug or a structurally-related compound after an initial hepatotoxicity event is common and may have serious consequences, particularly in hepatocellular-type toxicity. Careful history taking and reflecting diagnostic suspicion in the initial episode's record may reduce the incidence of this iatrogenic event.
\end{abstract}

Key words: Reexposure. Hepatotoxicity. Drug-induced liver disease. Hepatic adverse events. 
Fernández-Castañer A, García-Cortés M, Lucena MI, Borraz Y, Peláez G, Costa J, Anzola S, Salmerón J, Ávila S, Durán JA, Malcón de Dios A, Romero-Gómez M, Madrazo A, Muñoz-Yagüe T. Andrade RJ. Análisis de las causas, características y consecuencias de la reexposición al fármaco o compuesto responsable de un episodio de hepatotoxicidad. Rev Esp Enferm Dig 2008; 100: 278 -284.

\section{INTRODUCCIÓN}

La hepatotoxicidad, definida como la lesión o daño hepático causado por la exposición a un medicamento $\mathrm{u}$ otros agentes no farmacológicos, tiene especial trascendencia por su potencial gravedad, constituyendo una de las causas más frecuentes de retirada de medicamentos en Europa y en Estados Unidos (1,2). Aunque no existen figuras consistentes de incidencia y prevalencia de los efectos adversos hepáticos, se estima que la causa tóxica supone entre el 4-10\% de los casos de ictericia ingresados en un hospital general (3-5), pero la mayoría de los casos en un estudio reciente eran debidos a intoxicación con paracetamol, representando los casos debidos a hepatotoxicidad idiosincrásica únicamente un $0,7 \%$ (5). Un estudio prospectivo poblacional realizado en una región francesa estimó una tasa de incidencia anual de 14 casos por 100.000 habitantes de toxicidad hepática, con una incidencia estandarizada global de 80 casos por millón de habitantes y año (6). Los agentes antibacterianos, los antiinflamatorios no esteroideos y los analgésicos encabezan la lista de grupos terapéuticos incriminados con mayor frecuencia en las series de casos publicadas (7).

Dado que no existen marcadores específicos que nos permitan confirmar la existencia de hepatotoxicidad, el diagnóstico puede llegar a ser muy difícil y requiere una alta sospecha clínica por parte del médico (8). Para su detección, es imprescindible realizar una historia clínica exhaustiva, descartar otras causas de lesión hepática y conocer los factores de riesgo genéricos al desarrollo de hepatotoxicidad. Uno de los criterios más importantes para el diagnóstico de hepatotoxicidad es la recidiva de la lesión hepática tras la readministración (rechallenge) del fármaco sospechoso de producir lesión hepática, considerado por algunos autores como patrón oro para el diagnóstico de hepatotoxicidad (9). Esta readministración positiva viene definida por un incremento del doble del límite superior de la normalidad de los valores de ALT (lesión hepatocelular) y FA (lesión colestásica) tras la reexposición al fármaco.

Sin embargo, la realización de dicha prueba de readministración del fármaco imputado en un episodio de hepatotoxicidad con fines diagnósticos no está indicada por motivos éticos y además puede dar lugar a un falso negativo (10). Por lo tanto, únicamente estaría justificado realizar una prueba de reexposición dirigida en casos excepcionales donde el medicamento imputado es esencial, habiendo explicado al paciente los riesgos que conlleva y tras obtener su consentimiento por escrito. El presente estudio supone el primer análisis en la literatura de una serie amplia de casos con evidencia de reexposición positiva en relación con las circunstancias de la reexposición, variables demográficas, tipo de fármacos, duración e intervalo de tratamiento, indicaciones, tipos de lesión hepática, presencia de manifestaciones de hipersensibilidad, enfermedades concomitantes, factores de riesgo y evolución.

\section{MATERIAL Y MÉTODOS}

Los datos utilizados pertenecen al Registro Español de Hepatopatías Asociadas a Medicamentos, fundado en 1994 y coordinado por dos de los autores (R. J. Andrade y M. I. Lucena). Se identificaron los pacientes del registro con una reexposición positiva, definida como el incremento del doble de los valores de ALT y FA para los casos hepatocelulares y colestásicos/mixtos respectivamente tras la readministración del fármaco. Algunos de los casos incluidos en el presente estudio han sido previamente publicados como casos clínicos o series de casos (11-16).

La metodología de funcionamiento del registro, la sistemática de recogida de información y la evaluación de causalidad han sido previamente publicadas en detalle $(13,16)$. Para la recogida de datos se utilizó un protocolo estructurado que contiene los siguientes códigos: la relación temporal entre el comienzo del consumo del fármaco o la exposición al tóxico y el inicio de la enfermedad hepática y entre la suspensión del agente sospechoso y la mejora o recuperación de la disfunción hepática; descartar otras enfermedades hepáticas; presencia de factores de riesgo conocidos de hepatotoxicidad como el consumo de alcohol (cantidad en conversión del volumen de bebida en gramos) o embarazo; y el resultado del daño hepático. Se revisaron exhaustivamente los fármacos de consumo actual y los previos, productos de herboristería y posibles tóxicos. Se excluyeron otras causas de enfermedad hepática: hepatitis viral reciente por virus A ( $\operatorname{IgM}$ anti-VHA), B (IgM anti-VHB) o C (anti-VHC y ARN + por PCR), enfermedades autoinmunes (ANA, anticuerpos antimitocondriales y antimúsculo liso) y obstrucción biliar (ecografía abdominal complementada con resonancia magnética o colangiografía endoscópica en los casos que lo precisaban). Si el contexto clínico lo sugería, se descartó citomegalovirus, Epstein-Barr, infección por herpes virus o hepatitis E; y serología de bacterias como Salmonella, Campylobacter y Listeria. Si el paciente era $<$ de 40 años se determinó la ceruloplasmina y la excreción urinaria de cobre para descartar enfermedad de Wilson. Se evaluó asimismo la presencia de otras enfermedades metabólicas hepáticas como la hemocromatosis, el 
déficit de alfa 1 antitripsina y, en pacientes con historia de hipotensión reciente, se descartó la presencia de hepatitis isquémica. En casos dudosos, como pacientes con marcadores de autoinmunidad positivos, alcoholismo, enfermedad hepática previa o enfermedades sistémicas que pueden afectar al hígado, se indicó una biopsia hepática para obtener más información etiológica.

Los casos fueron revisados por el médico responsable y posteriormente evaluados por tres expertos independientes del centro coordinador, quienes valoran la causalidad, primero por juicio clínico y posteriormente mediante la aplicación de la escala propuesta por el Council for International Organizations of Medical Sciences, (CIOMS) también denominada Roussel-Uclaff Causality Assessment Method (RUCAM) (17,18). Dicha escala se basa en un sistema de puntuación estandarizado según diferentes criterios (cronológicos, evolución, factores de riesgo, fármaco concomitante, exclusión de causas alternativas no farmacológicas, información previa de hepatotoxicidad del fármaco, reexposición), finalmente el resultado se traslada a categorías de sospecha divididas en: definida o altamente probable (> 8 puntos), probable (6-8 puntos), posible (3-5 puntos), improbable ( $<2$ puntos) y excluida ( $\leq 0$ puntos). Únicamente los casos valorados por la escala CIOMS como posible, probable o definido se incluyeron en la base de datos.

La definición y clasificación del tipo de lesiones fueron establecidas mediante la aplicación de los criterios derivados de la reunión del Grupo Internacional de Consenso (19). Cuando las alteraciones bioquímicas tras la readministración no llegaron a elevarse a más del doble del límite superior de la normalidad, se consideró como alteración biológica. Se definió lesión hepática al incremento de dos veces sobre el límite superior a la normalidad (LSN) en alanino aminotransferasa (ALT) o bilirrubina conjugada; o a la combinación del incremento de aspartato aminotransferasa (AST), fosfatasa alcalina (FA) y bilirrubina total siendo uno de ellos 2 veces > LSN. Se clasificó el tipo de lesión en base a los hallazgos anatomopatológicos o alternativamente en base a los hallazgos bioquímicos si no se disponía de biopsia. El daño hepático se determinó como hepatocelular cuando el ratio ALT/FA era $>5$, como colestásica $<2$ y mixta $>2$ pero $<5$. Se utilizaron para dicha clasificación los primeros valores analíticos realizados al paciente. Finalmente se intentó establecer el mecanismo patogénico del daño hepático, clasificándolo en intrínseco o idiosincrásico, y este último a su vez en inmunoalérgico si presentaba datos de hipersensibilidad como fiebre, exantema y presencia de eosinófilos en sangre o en la biopsia hepática.

Los fármacos responsables de la reacción hepática se clasificaron de acuerdo con el Anatomical Therapeutic Classification (ATC) recomendado por WHO-Europe (20).

Los datos obtenidos fueron analizados con el paquete estadístico SPSS (Statistical Package for the Social Sciences, versión 10.0 para Windows). Se realizó un análisis descriptivo y se aplicó el test de Chi cuadrado. Las diferencias fueron consideradas estadísticamente significativas si el valor de $p$ era menor de 0,05 .

\section{RESULTADOS}

De un total de 520 casos de hepatotoxicidad del Registro Regional de Hepatotoxicidad, en 33 casos había historia de reexposición a la sustancia responsable de un episodio previo. De estos, 2 pacientes no cumplieron los criterios de reexposición positiva, dado que se consideraron como alteración biológica y fueron excluidos del análisis. Un total de 31 casos (6\%) cumplieron, pues, los criterios definidos. En la tabla I se muestran las principales características demográficas y clínicas de los pacientes, incluidos en el registro, con episodios previos de hepatotoxicidad y readministración, bien del fármaco imputado o de otro perteneciente al mismo grupo farmacológico.

Veintidós pacientes $(71 \%)$ de la serie presentaron una lesión de tipo hepatocelular y en nueve (29\%) el daño fue de tipo colestásico o mixto. En la tabla II se muestra la descripción de las distintas variables tanto cualitativas como cuantitativas en relación con los distintos tipos de lesión hepática de los casos con reexposición positiva. La edad media global de la serie fue de 42 años (42 años en el grupo hepatocelular y de 47 años en el grupo colestásico/mixto). El $48 \%$ eran del sexo masculino ( $45 \%$ en el grupo hepatocelular y $55 \%$ en el grupo colestásico/mixto).

La duración media del tratamiento fue mayor en el tipo de lesión hepatocelular, 100 vs. 87 días. Dicha duración fue menor en el segundo episodio tras la reexposición en ambos tipos de lesión hepática (Tabla II). Doce pacientes (39\%) presentaron datos de hipersensibilidad (eosinofilia, rash o fiebre). Los hallazgos bioquímicos fueron como siguen: la ALT media fue de $998 \mathrm{UI} / \mathrm{dl}$ en el primer episodio y de 812 en la reexposición; los resultados de la fosfatasa alcalina fueron de $275 \mathrm{UI} / \mathrm{dl}$ al inicio y de $265 \mathrm{UI} / \mathrm{dl}$ en el segundo episodio; la bilirrubina media fue de $11,09 \mathrm{mg} / \mathrm{dl}$ en la primera administración del fármaco y de 6,31 mg/dl en la readministración.

En cuanto a la gravedad del cuadro, 16 pacientes requirieron ingreso hospitalario (52\%), 12 de los cuales presentaron lesión hepatocelular $(55 \%)$ y cuatro desarrollaron daño colestásico/mixto (44\%). Tres de los pacientes ingresados con lesión de tipo hepatocelular presentaron una evolución fatal, 2 de los cuales fallecieron debido a insuficiencia hepática y un tercero se derivó a un hospital con programa de trasplante debido al desarrollo de un fallo hepático fulminante. En el grupo colestásico/mixto se produjo también un caso de fallo hepático agudo que precisó la realización de un trasplante hepático.

Se realizó biopsia hepática percutánea en 11 pacientes de la serie cuyos hallazgos se recogen en la tabla I. La lesión histológica más frecuente fue la hepatitis colestásica en 5 casos, seguida de necrosis hepatocelular en 4 pacientes. En un caso hubo necrosis centrozonal. Finalmente, la biopsia realizada al paciente con sospecha de hepatotoxicidad secundaria a estradiol dio como resultado cambios inespecíficos.

El grupo farmacológico identificado con mayor frecuencia fue el de los antiinfecciosos con 8 casos (26\%), seguido 
Tabla I. Datos demográficos y clínicos de los casos con hepatotoxicidad y reexposición positiva incluidos en el registro de hepatotoxicidad

\begin{tabular}{|c|c|c|c|c|c|c|c|c|c|c|c|c|c|c|}
\hline $\begin{array}{l}\text { Edadl } \\
\text { Sexo }\end{array}$ & Fármaco & $\begin{array}{l}\text { Latencia } \\
I^{\prime \prime} \text { epis. } \\
\text { (dias) }\end{array}$ & $\begin{array}{l}\text { Latencia } \\
2^{\circ} \text { epis. } \\
\text { (dias) }\end{array}$ & $\begin{array}{c}\text { Duración } \\
1^{e r} \text { epis. } \\
\text { (dias) }\end{array}$ & $\begin{array}{c}\text { Duración } \\
2^{0} \text { epis. } \\
\text { (dias) }\end{array}$ & $\begin{array}{c}B T \\
m g / d l\end{array}$ & $\begin{array}{l}\text { ALT1 } \\
(\text { LSN) }\end{array}$ & $\begin{array}{l}\text { ALT2 } \\
\text { (LSN) }\end{array}$ & $\begin{array}{l}\text { FA1 } \\
(\text { ISN) }\end{array}$ & $\begin{array}{l}F A 2 \\
(L S N)\end{array}$ & $\begin{array}{l}\text { Tipo } \\
\text { daño }\end{array}$ & Biopsia & CIOMSIRUCAM & Comentarios \\
\hline $23 / \mathrm{F}$ & Mesalacina & 210 & & 190 & 275 & 1 & 4,2 & 4,7 & 0,55 & 0,6 & $H C$ & & Altamente probable (9) & Hospitalización \\
\hline $33 / \mathrm{M}$ & Lansoprazol (11) & 58 & & 58 & & & 6 & 2,5 & 1,2 & - & $H C$ & & Altamente probable (10) & \\
\hline $43 / F$ & Fluoxetina & 180 & & 185 & & & 1,7 & - & - & - & & & No aplicable & Alt. biológica. RC paroxetina (HTX previa) \\
\hline $17 / \mathrm{F}$ & Clomifeno & 47 & & 137 & & 0,3 & 5,4 & 29,6 & 0,9 & - & $H C$ & & Probable (8) & \\
\hline 30/F & Paracetamol (12) & 18 & 1 & 18 & 1 & 14 & 26,3 & 7,1 & 5,1 & - & $H C$ & & Altamente probable (11) & Hospitalización, HS \\
\hline 34/M & Kava-kava & 150 & & 152 & & 0,5 & 18 & 1,4 & 1,2 & 0,7 & $H C$ & & Probable (6) & HS \\
\hline $32 / \mathrm{F}$ & Isoniacida & 1 & & 6 & 14 & 6,7 & 5,8 & 11,7 & 3,3 & 6,6 & $\mathrm{COL}$ & & Probable (8) & $H S$ \\
\hline $41 / M$ & Amoxi.clav. (13) & 35 & & 5 & 4 & 10 & 34,3 & 18,6 & 1,1 & 3 & & Hepatitis colestásica & Altamente probable (9) & Hospitalización, THO, HS \\
\hline $23 / F$ & Camellia sinensis (14) & 19 & & & 21 & 11 & 31,1 & 71,1 & 2,5 & 1,01 & & Necrosis hepatocelular & Altamente probable (11) & Hospitalización, FHF, HS \\
\hline $43 / F$ & Amineptina & 420 & 1 & 420 & 1 & 3,6 & 14,3 & - & 1 & - & $H C$ & & Altamente probable (9) & Hospitalización, exitus, autolisis \\
\hline $61 / M$ & Eritromicina & & 8 & & 1 & 2,2 & 5,3 & - & 1,7 & - & MIX & & Altamente probable (11) & HS \\
\hline $25 / \mathrm{M}$ & Amoxi.-clav. (13) & 23 & 1 & 14 & 1 & 7,4 & 6,4 & 34,2 & 1,4 & 0,5 & HC & & Altamente probable (10) & Hospitalización, HS \\
\hline $73 / \mathrm{M}$ & Clometiazol (15) & 40 & 15 & 90 & 15 & 3,8 & 22,2 & 25,3 & 2,7 & 3,4 & $H C$ & & Altamente probable (12) & Hospitalización \\
\hline 49/M & Azitromicina & & 10 & 3 & 15 & 6,6 & - & 51,5 & - & 3 & $H C$ & & Altamente probable(11) & Hospitalización \\
\hline $41 / \mathrm{F}$ & Bentazepam & & 180 & 330 & 185 & 0,4 & - & 7,4 & - & 1 & & H. crónica activa & Probable (7) & \\
\hline $46 / F$ & Irrbersartán & 234 & & 274 & 82 & 3,3 & 44,3 & 34,9 & 1,4 & 1 & & Necrosis hepatocelular & Probable (8) & Hospitalización \\
\hline $52 / M$ & Azatioprina & & 8 & 14 & 10 & 7,5 & 2,2 & - & 7,1 & - & $\mathrm{COL}$ & & Altamente probable (11) & HS \\
\hline $27 / \mathrm{F}$ & Estradiol & 190 & & 195 & & 0,6 & 5,1 & 2,4 & 1,1 & 1,1 & MIX & Inespećfíco & Probable (6) & \\
\hline $25 / \mathrm{M}$ & Interferón & 6 & & 7 & 121 & 1,8 & 27,8 & 15,1 & 0,9 & 1,8 & HC & & Altamente probable (13) & \\
\hline 46/F & Propafenona & 0 & & 2 & 6 & 3,4 & 38,9 & - & 0,9 & - & $H C$ & & Altamente probable (10) & HS \\
\hline $71 / M$ & Pirazinamida & & 5 & & 16 & 0,66 & 13,1 & - & 1,1 & - & HC & & Altamente probable (12) & Hospitalización, exitus \\
\hline $26 / F$ & Camellia sinensis & 121 & 45 & 121 & & 17 & 43,8 & 46,4 & 0,8 & 1,1 & & Necrosis hepatocelular & Altamente probable $(9)$ & Hospitalización, HS \\
\hline 48/M & Amiodarona & 4 & & 3 & & 2,5 & 73,8 & - & 0,8 & - & $H C$ & & Altamente probable (10) & Hospitalización \\
\hline $24 / F$ & Ácido valproico & & 1 & 20 & 23 & 5,2 & - & 60,5 & - & 0,5 & & Hepatitis colestásica & Altamente probable (11) & Hospitalización \\
\hline $16 / \mathrm{M}$ & Amoxi-clav. & 14 & & 15 & 30 & 1,4 & 14,6 & 4,6 & 1,8 & 1,9 & $H C$ & & Altamente probable (10) & HS \\
\hline $44 / F$ & Tamoxifeno & 330 & & 335 & 60 & 1,8 & 112 & & 87 & & & Hepatitis colestásica con fibrosis y cirrosis & Altamente probable(12) & Hospitalización \\
\hline 46/M & Ebrotidina (16) & 20 & 1 & 30 & 1 & 7 & 65,9 & 13,1 & 1,69 & - & & Necrosis zonal & Altamente probable (11) & \\
\hline $61 / F$ & Fluvastatina & & 1 & & 1 & 4,2 & - & 4,9 & - & 2,5 & $H C$ & & Altamente probable (9) & \\
\hline 79/M & Ciproterona & & 77 & 93 & 77 & 16 & 10,2 & 8,7 & 0,6 & 0,6 & & Hepatitis colestásica & Altamente probable (10) & Hospitalización \\
\hline 48/M & Fluvastatina & & 1 & 30 & 1 & 11 & 4,2 & - & 1 & - & & Hepatitis colestásica & Altamente probable (9) & \\
\hline $77 \mathrm{M}$ & Metotrexato & & & 1.291 & & 0,9 & 1,07 & - & 0,3 & - & - & & No aplicable & Alt. biológica \\
\hline $52 / F$ & Diclofenaco & 23 & & 23 & 38 & 9,8 & 62,5 & 57,8 & - & 1,6 & $H C$ & & Probable (8) & Hospitalización, HS \\
\hline $69 / F$ & Amoxi-clav. & & 4 & 14 & 8 & 3,15 & 20,9 & 10,6 & 2,6 & 1,4 & $H C$ & & Altamente probable (10) & \\
\hline
\end{tabular}

ALT: alanino aminotransferasa; AMA: anticuerpos antimitocondriales; AML: anticuerpos antimúsculo liso; ANA: anticuerpos antinucleares; AP: anatomía patológica; AST: aspartato aminotransferasa; Br: bilirrubina total; Col: colestásico F: femenino; FA: fosfatasa alcalina; FHF: fallo hepático fulminante; HC: hepatocelular ; HS: hipersensibilidad;; M: masculino; LSN: límite superior a la normalidad; Mix: mixto; VHC: virus de la hepatitis C; VIH: virus de la inmunodeficiencia humana.

por los fármacos del sistema nervioso y los cardiovasculares con 5 casos respectivamente (16\% cada grupo). El fármaco más frecuentemente implicado fue el antibiótico amoxicilina-ácido clavulánico. Tres casos de la serie se debieron a fármacos nuevos con menos de tres años desde su comercialización cuando se produjo la reacción, uno de ellos debido a ebrotidina, que tuvo que ser retirada del mercado debido a hepatotoxicidad $(11,16)$.

Las circunstancias en que ocurrió la reexposición y los fármacos responsables del cuadro se recogen en la tabla III, donde se incluyen también los dos casos con readministración del fármaco responsable de un episodio previo de hepatotoxicidad, pero que no elevaron las enzimas hepáticas a más del doble del límite superior a lo normal. Como puede apreciarse, en 24 casos $(73 \%)$ la reexposición fue accidental y las circunstancias en las que ocurrió fueron variables. Las causas fundamentales de esta readministración accidental fueron la ausencia de diagnóstico definitivo o sospecha de hepatotoxicidad en el primer episodio en 21 casos (64\% del total) y la falta de información al paciente o los facultativos responsables del mismo en tres casos (9\% del total). Por otro lado, cinco episodios (15\%) se produjeron en pacientes con patologías en las que el fármaco imputado en la reacción era imprescindible o la mejor alternativa terapéutica para el paciente y 4 casos (12\%) en los que el fármaco que provocó la reexposición no fue el mismo del episodio inicial (sí del mismo grupo farmacológico) presumiblemente debido al desarrollo de una reacción cruzada.

\section{DISCUSIÓN}

En el presente estudio se identificaron 33 pacientes con historia de readministración de un fármaco imputado previamente en un episodio de toxicidad hepática de 520 casos recogidos en el Registro Español de Hepatotoxicidad en el momento del estudio. De estos, 31 pacientes 
Tabla II. Datos clínicos y resultados analíticos de los casos de readministración positiva según el tipo de hepatotoxicidad

\begin{tabular}{|c|c|c|c|}
\hline & $\begin{array}{l}\text { Hepatocelular } \\
\quad(n=22)\end{array}$ & $\begin{array}{l}\text { Col./mix. } \\
(n=9)\end{array}$ & $\begin{array}{c}\text { Total } \\
(n=31)\end{array}$ \\
\hline Edad & $41(16-71)$ & $47(25-77)$ & 42 \\
\hline Sexo (\% hombres) & $45 \%$ & $55 \%$ & $48 \%$ \\
\hline \multicolumn{4}{|l|}{ Duración (días) } \\
\hline $1^{\mathrm{er}}$ tratamiento & $100(2-330)$ & $87(5-335)$ & 93 \\
\hline $2^{\circ}$ tratamiento & $48(1-275)$ & $29(1-77)$ & 40 \\
\hline Días de aparición & $24(1-420)$ & $70(1-330)$ & 76 \\
\hline Ictericia & $68 \%$ & $68 \%$ & \\
\hline Datos de hipersensibilidad & $36 \%$ & $44 \%$ & $39 \%$ \\
\hline \multicolumn{4}{|l|}{$\mathrm{BT}(\mathrm{mg} / \mathrm{dl})$} \\
\hline $2^{\circ}$ episodiodio & $11,1(0,7-21,0)$ & $3,9(0,6-7,5)$ & 11,09 \\
\hline $\begin{array}{l}2^{\circ} \text { episodio } \\
\text { ALT (UI/dl) }\end{array}$ & $6,38(0,7-16,6)$ & $6,1(0,5-11)$ & 6,31 \\
\hline \multicolumn{4}{|l|}{$\operatorname{ALT}(\mathrm{Ul} / \mathrm{dl})$} \\
\hline $1^{\text {er }}$ episodio & $1.107(437-2.252)$ & $162(43-258)$ & 998 \\
\hline $2^{\circ}$ episodio & $1.016(57-2.722)$ & $185(47-468)$ & 812 \\
\hline \multicolumn{4}{|l|}{ GGT (U//dl) } \\
\hline $1^{\text {er }}$ episodio & $225(62-587)$ & $526(55-1.769)$ & 225 \\
\hline $2^{\circ}$ episodio & $202(44-743)$ & $263(17-492)$ & 209 \\
\hline \multicolumn{4}{|l|}{ FA } \\
\hline $1^{\text {er }}$ episodio & $275(74-661)$ & $310(71-910)$ & 275 \\
\hline $2^{\circ}$ episodio & $259(70-815)$ & $315(61-848)$ & 265 \\
\hline Tiempo de cese (días) & $86(7-420)$ & $74(14-300)$ & 20 \\
\hline Hospitalización & $12(55 \%)$ & $4(44 \%)$ & $16(52 \%)$ \\
\hline Evolución fatal (muerte o THO) & $3(14 \%)$ & $1(11 \%)$ & $4(13 \%)$ \\
\hline
\end{tabular}

cumplieron los criterios de reexposición positiva, lo que constituye un $6 \%$ del total del registro, cifra no despreciable debido a su impacto potencial y al hecho de que debería ser prevenible en la mayoría de los casos.

Entre los episodios de reexposición, predominó el tipo de daño hepatocelular, mostrando, asimismo, un peor pronóstico con un mayor número de ingresos y más frecuente evolución a trasplante o muerte. Estos hallazgos son acordes a los resultados obtenidos del análisis global de la totalidad del registro (7). Sí hubo, en cambio, un mayor porcentaje de manifestaciones de hipersensibilidad (39\%) en este grupo de pacientes comparado con el correspondiente al análisis global del registro, donde se detectaron signos de inmunoalergia en el $23 \%$ de los casos (13). Además, aun considerando el reducido número de pacientes con reexposición en el grupo colestásico y mixto, la frecuencia de casos con datos de inmunoalergia fue elevada, lo que sugiere que dicho mecanismo patogénico se expresa más comúnmente con un patrón de lesión colestásico/mixto. Merece destacarse asimismo que, aunque se realizó una biopsia hepática en un tercio de los pacientes de la serie, los hallazgos no fueron en general concluyentes para el diagnóstico de hepatotoxicidad, ya que el propio episodio de reexposición tenía un mayor peso. En circunstancias de reexposición la biopsia podría tener, pues, una utilidad principalmente de tipo pronóstico. De hecho, en uno de estos episodios debido a bentazepam la biopsia fue consistente con una hepatitis cróni-
Tabla III. Causas de reexposición y los fármacos responsables del mismo

\begin{tabular}{|c|c|}
\hline Causas de readministración & Fármacos \\
\hline $\begin{array}{l}\text { Accidental (73\%) } \\
\text { Ausencia de diagnóstico de hepatotoxicidad en el } \\
\text { Ter episodio }^{\text {Retiran el fármaco sin sospechar HTX }}\end{array}$ & $\begin{array}{l}\text { Propafenona } \\
\text { Clormetiazol } \\
\text { Amoxi-clav.: } 4 \text { casos } \\
\text { Azitromicina }\end{array}$ \\
\hline Fármaco nuevo. No HTX descrita previamente & $\begin{array}{l}\text { Ebrotidina } \\
\text { Irbesartán }\end{array}$ \\
\hline Productos de herboristería & $\begin{array}{l}\text { Kava Kava } \\
\text { C. sinensis: } 2 \text { casos }\end{array}$ \\
\hline Caso atípico de HTX & Paracetamol \\
\hline $\begin{array}{l}\text { Múltiples fármacos posibles, imputación del caso } \\
\text { índice a otro fármaco }\end{array}$ & $\begin{array}{l}\text { Diclofenaco } \\
\text { Pirazinamida }\end{array}$ \\
\hline $\begin{array}{l}\text { No importancia adecuada al incremento de } \\
\text { transaminasas en el } 1^{\text {er }} \text { episodio }\end{array}$ & $\begin{array}{l}\text { Amiodarona } \\
\text { Clomifeno } \\
\text { Azatioprina }\end{array}$ \\
\hline $\begin{array}{l}\text { Manifestaciones inespecíficas en el } 1^{\text {er }} \text { episodio } \\
\text { Retirada por intolerancia }\end{array}$ & $\begin{array}{l}\text { Mesalacina } \\
\text { Lansoprazol (nuevo) } \\
\text { Fluvastatina }\end{array}$ \\
\hline $\begin{array}{l}\text { Falta de información al paciente, no información en } \\
\text { informe clínico o cambio de médico }\end{array}$ & $\begin{array}{l}\text { Amineptina } \\
\text { Ácido valproico } \\
\text { Eritromicina }\end{array}$ \\
\hline Reacción cruzada (12\%) & $\begin{array}{l}\text { Ciproterona vs. flutamida } \\
\text { Fluvastatina vs. pravastatina } \\
\text { Fluoxetina vs. paroxetina } \\
\text { Paroxetina vs. bentazepam }\end{array}$ \\
\hline Tratamiento imprescindible o mejor alternativa (15\%) & $\begin{array}{l}\text { Isoniazida } \\
\text { Metotrexato } \\
\text { Tamoxifeno } \\
\text { Interferón alfa 2beta } \\
\text { Estradiol }\end{array}$ \\
\hline
\end{tabular}

HTX: hepatotoxicidad.

ca activa, como ha sido previamente descrito con este fármaco (21), y que incrementa el riesgo de persistencia de la lesión hepática $(22,23)$. La indicación de la biopsia fue, en todo caso, más frecuente en la lesión hepatocelular, probablemente debido a que este tipo de lesión es más inespecífica, siendo más difícil su vinculación con la etiología tóxica (23). Es importante señalar, asimismo, que el periodo de latencia fue menor tras la reexposición que en el primer episodio.

El grupo terapéutico más frecuentemente implicado en los episodios de reexposición fue el de los antibióticos, no difiriendo este hallazgo con lo descrito en la valoración general del Registro de Hepatotoxicidad (7). Este hecho refleja que tal acontecimiento no está únicamente vinculado a un grupo terapéutico específico, sino que es también la consecuencia de las condiciones de uso de los medicamentos $(7,15)$. 
Analizando las circunstancias en las que ocurrió la reexposición, se evidenció una mayor frecuencia de readministración de tipo accidental debido a la falta de sospecha o diagnóstico definitivo de hepatotoxicidad (bien sea por tratarse de un fármaco nuevo o un producto de herboristería), no dar importancia a un aumento de transaminasas en la primera exposición, falta de información al paciente o el facultativo responsable del mismo o ser una expresión de hepatotoxicidad inusual para el fármaco en cuestión. Ejemplo de esto último fue el caso de hepatotoxicidad secundaria a paracetamol de esta serie, el cual fue de naturaleza idiosincrásica con signos de hipersensibilidad, algo excepcional que hizo difícil el diagnóstico (12).

Otra circunstancia evidenciada en este estudio fue la incapacidad para identificar o imputar la enfermedad hepática al fármaco responsable del primer episodio, en especial cuando se administraron varios compuestos con potencial hepatotóxico. Un ejemplo de este hecho fue el caso del diclofenaco, en el que el primer episodio de lesión hepática fue imputado al antibiótico amoxicilinaácido clavulánico administrado simultáneamente al antiinflamatorio, lo cual no impidió la readministración del fármaco culpable con la consiguiente recidiva de la enfermedad.

Un hecho sorprendente y evitable, que ocurrió en tres casos de esta serie y que determinó la readministración no intencional del fármaco responsable, fue la falta de información sobre el primer episodio a los pacientes, su médico de cabecera o su constatación en el informe clínico. Como ejemplo destacar el caso de toxicidad hepática inducida por ácido valproico, en el que el paciente cambió de médico y, al no reflejarse en el informe el primer episodio, se prescribió nuevamente dicho antiepiléptico por parte del segundo facultativo con la consiguiente recidiva del cuadro.

Finalmente, hay que señalar que en cuatro casos el fármaco responsable del segundo episodio fue diferente del causante del cuadro inicial. Ello pone de manifiesto la necesidad de estar advertido de la posibilidad de reacciones cruzadas de hepatotoxicidad con fármacos estructuralmente relacionados (7).

Curiosamente, la reexposición deliberada del paciente al fármaco responsable, por ser este la única o mejor alternativa para su enfermedad de base (circunstancia que sería una de las escasas indicaciones de reexposición intencionada éticamente aceptables), sólo representó el $15 \%$ en esta serie.

Las recomendaciones prácticas que podrían derivarse de los resultados de este estudio serían la importancia de la información al paciente, al facultativo responsable del mismo y la elaboración de un informe clínico detallado en los casos en que se haya detectado una reacción hepatotóxica con el fin de evitar confusiones ante cambio de médico. Por otro lado, se debería estimular la notificación de las reacciones hepatótoxicas y extremar la vigilancia ante el uso de fármacos nuevos y productos de herboristería. Por último, el clínico debe estar advertido sobre la posibilidad de aparición de reacciones cruzadas entre distintos medicamentos.

Como conclusión, podemos afirmar que las consecuencias de una lesión hepatotóxica por reexposición al fármaco imputado en un episodio previo de daño hepático o compuestos estructuralmente similares pueden ser potencialmente graves, precisando en algunos pacientes la hospitalización, trasplante hepático e incluso pudiendo llegar a producirse la muerte del enfermo. Si bien es cierto que es difícil llegar al diagnóstico por la ausencia de pruebas específicas, una alta sospecha clínica inicial, un estudio concienzudo y una información clara y detallada al paciente y su médico son fundamentales para prevenir la readministración accidental de fármacos imputados en casos de lesión hepática inducida por medicamentos.

\section{AGRADECIMIENTOS Y FINANCIACIÓN}

Agradecemos la participación de D. Ramón Hidalgo en el análisis estadístico de los datos. Este estudio ha sido parcialmente financiado con una ayuda de la Agencia Española del Medicamento y con una beca FIS 07/0980.

\section{GRUPO ESPAÑOL DE ESTUDIO DE HEPATOPATÍAS ASOCIADAS A MEDICAMENTOS}

\section{Centros clínicos participantes}

Hospital Universitario Virgen de la Victoria, Málaga (centro coordinador): R. J. Andrade, M. I. Lucena, Y. Borraz, M. García-Cortés, E. Ulzurrun, S. López-Ortega, M. Robles, K. Patchkoria, J. L. López-Durán, E. LópezTorres, C. Verge, R. Camargo, E. García-Ruiz, E. Fernández, R. Alcántara, R. Cueto, F. Bermúdez.

Hospital Torrecárdenas, Almería: M. C. Fernández, G. Peláez, R. Daza, M. Casado, J. L. Vega, F. Suárez, M. Torres, M. González-Sánchez, J. Esteban.

Hospital Universitario Virgen de Valme, Sevilla: M. Romero, A. Madrazo, R. Corpas, E. Suárez.

Hospital de Mendaro, Guipuzkoa: A. Castiella, E. M. Zapata.

Hospital Germans Trias i Puyol, Barcelona: R. Planas, S. Anzola, J. Costa, N. López, F. García-Góngora, A. Barriocanal, A. Borras, E. Gallardo, A. Vaqué, A. Soler.

Hospital Costa del Sol, Málaga: J. M. Navarro, J. F. Rodríguez.

Hospital Central de Asturias, Oviedo: R. Pérez-Álvarez, L. Rodrigo-Sáez.

Hospital Universitario San Cecilio, Granada: J. Salmerón, A. Gila, V. Bellot, A. Caballero.

Hospital Universitario Virgen de las Nieves, Granada: R. Martín-Vivaldi, F. Nogueras.

Hospital Carlos Haya, Málaga: M. Jiménez.

Hospital Sant Pau, Barcelona: C. Guarner, E. M. Román, G. Soriano. 
Hospital Puerta del Mar, Cádiz: F. Díaz, M. J. Soria, L. Martín-Herrera, P. Rendón, M. Macías.

Hospital Virgen de la Macarena, Sevilla: A. Malcón de Dios, J. A. Durán, M. Jiménez-Sáez, A. Ruiz, J. Alanis-López, M. Villar.

Hospital Morales Meseguer, Murcia: H. Hallal Hachem.

Hospital de Puerto Real, Cádiz: J. M. Pérez-Moreno, M. Puertas.

Hospital La Inmaculada. Huércal-Overa, Almería: $\mathrm{H}$. Sánchez-Martínez.

Hospital Virgen del Rocío, Sevilla: J. Aguilar, S. Otero, F. Reina, A. Pizarro.

Hospital Juan Ramón Jiménez, Huelva: M. Ramos, T. Ferrer.

Hospital Ciudad de Jaén: E. Baeyens.

Hospital de Osuna, Sevilla: J. Pérez-Martínez.

Hospital 12 de Octubre, Madrid: T. Muñoz-Yagüe, J.

A. Solís-Herruzo.

Hospital General Básico de Vélez, Málaga: F. Santa-

lla, C. Sánchez-Robles.

Hospital Marqués de Valdecilla, Santander: F. Pons,

R. Taheri.

Hospital de Ronda, Málaga: V. Díaz-Morán.

Hospital Xeral-Calde, Lugo: S. Ávila-Nasi.

Hospital de Donosti, San Sebastián: M. García-Bengoechea.

Hospital de Basurto, Bilbao: S. Blanco, P. Martínez.

Hospital del Mar, Barcelona: R. Solá.

Hospital Comarcal de Antequera, Málaga: F. Cárdenas.

Hospital General Universitario Gregorio Marañón,

Madrid: R. Bañares.

Hospital General de Valencia: M. Diago.

Hospital de Sagunto, Valencia: J. Primo, J. R. Molés.

Hospital Clínico Universitario Miguel Servet, Zaragoza:

M. A. Simón.

Hospital de Laredo, Cantabria: M. Carrasco.

Hospital Clínic, Barcelona: M. Bruguera.

\section{BIBLIOGRAFÍA}

1. Navarro VJ, Senior JR. Drug-related hepatotoxicity. N Engl J Med 2006; 354: 731-9.

2. Bakke OM, Manocchia M, De Abajo F, Kaitin KI, Lasagna L. Drug safety discontinuations in the United Kindom, the United States, and Spain from 1974 through 1993: A regulatory perspective. Clin Pharmacol Ther 1995; 58: 108-17.

3. Whitehead MW, Hainsworth I, Kingham JGC. The causes of obvious jaundice in South West Wales: 2000. Gut 2001; 48: 409-13.

4. Björnsson E, Ismael S, Nejdet S, Kilander A. Severe jaundice in Swe- den in the new millennium: Causes, investigations, treatment and prognosis. Scand J Gastroenterol 2003; 38: 86-94.

5. Vuppalanchi R, Liangpunsakul S, Chalasani N. Etiology of new-onset jaundice: How often is it caused by idiosyncratic drug-induced liver injury in the United States? Am J Gastroenterol 2007; 102: 55862.

6. Sgro C, Clinard F, Ouazir K, Chanay H, Allard C, Guilleminet C, et al. Incidence of drug-induced hepatic injuries: A French populationbased study. Hepatology 2002; 36: 451-5.

7. Andrade RJ, Lucena MI, Fernández MC, Peláez G, Pachkoria K, García-Ruiz E, et al. Drug-induced liver injury: An analysis of 461 incidences submitted to the Spanish registry over a 10 -year period. Gastroenterology 2005; 129: 512-21.

8. Bissel DM, Gores GJ, Laskin DL, Hoofnagle JH. Drug-induced liver injury: Mechanisms and test systems. Hepatology 2001; 33: 1009-13.

9. Larrey D. Epidemiology and individual susceptibility to adverse drug reactions affecting the liver. Sem Liv Dis 2002; 22: 145-55.

10. Zimmerman HJ. Hepatotoxicity: The adverse effects of drugs and other chemicals on the liver. Philadelphia: Lipincot Willliams and Wilkins; 1999.

11. García-Cortés M, Lucena MI, Andrade RJ, Romero-Gómez M, Fernández C. Lansoprazole-induced hepatic dysfunction. Ann Pharmacother 2003; 37: 1731.

12. Andrade RJ, Lucena MI, García-Escaño MD, Camargo R. Severe idiosyncratic acute hepatic injury caused by paracetamol. J Hepatol 1998; 28: 1078.

13. Andrade RJ, Lucena MI, Fernández MC, Vega JL, Camargo R. Hepatotoxicity in patients with cirrhosis, an often unrecognized problem: Lessons from a fatal case related to amoxicillin/clavulanic acid. Dig Dis Sci 2001; 46: 1416-9.

14. Gavilán JC, Bermúdez FJ, Salgado F, Pena D. Fitoterapia y hepatitis. Rev Clin Esp 1999; 199: 693-4.

15. Gallardo-Caballero E, Lucena MI, Andrade RJ, Rodrigo L, PérezMartínez J, García-Ruiz E. Severe toxic hepatitis related to chlormethiazole. J Hepatol 2003; 39: 1092-3.

16. Andrade RJ, Lucena MI, Martín-Vivaldi R, Fernández MC, Nogueras F, Peláez G, et al. Acute liver injury associated with the use of ebrotidine, a new H2-receptor antagonist. J Hepatol 1999; 31: 641-6.

17. Danan G, Benichou C. Causality assessment of adverse reactions to drugs I. A novel method based on the conclusions of international consensus meeting: Application to drug-induced liver injures. J Clin Epidemiol 1993; 43: 1323-30.

18. Bénichou C, Danan G, Flahault A. Causality assessment of adverse reactions to drugs-II. An original model or validation of drug causality assessment methods: Case reports with positive rechallenge. J Clin Epidemiol 1993; 46: 1331-6.

19. Benichou C. Report of an International Consensus Meeting. Criteria of drug-induced liver disorders. J Hepatol 1990; 11: 272-6.

20. World Health Organization Collaborating Center for drugs statistics methodology. Anatomical Therapeutic Chemical (ATC) classification index including defined daily dose (DDDs) for plain substances. Oslo: World Health Organization Collaborating Center for drugs statistics methodology; 2002.

21. Andrade RJ, Lucena MI, Aguilar J, Lazo MD, Camargo R, Moreno P, et al. Chronic liver injury related with the use of bentazepam: An unusual instance of benzodiazepine hepatotoxicity. Dig Dis Sci 2000; 45: $1400-4$.

22. Andrade RJ, Lucena MI, Kaplowitz N, García-Muñoz B, Borraz Y, Pachkoria K, et al. Outcome of acute idiosyncratic drug-induced liver injury: Long-term follow-up in a hepatotoxicity registry. Hepatology 2006; 44: 1581-8.

23. Lucena MI, García-Cortés M, Cueto R, López-Durán JL, Andrade RJ. Assessment of drug-induced liver injury in clinical practice. Fund Clin Pharmacol 2008; in press. 